\title{
ASO Author Reflections: Effects of the Updated AJCC Classification for Patients with Stage III Melanoma
}

\author{
Dimitrios Katsarelias, $\mathrm{MD}^{1}$, Karolin Isaksson, $\mathrm{MD}^{2}$, and Roger Olofsson Bagge, $\mathrm{MD}^{1,3}$ \\ ${ }^{1}$ Department of Surgery, Institute of Clinical Sciences, Sahlgrenska Academy at the University of Gothenburg, Sahlgrenska \\ University Hospital, Gothenburg, Sweden; ${ }^{2}$ Department of Clinical Sciences Lund, Surgery, Lund University, Skåne \\ University Hospital, Lund, Sweden; ${ }^{3}$ Wallenberg Centre for Molecular and Translational Medicine, University of \\ Gothenburg, Gothenburg, Sweden
}

\section{PAST}

The incidence of cutaneous melanoma is steadily increasing in most countries with fair-skin populations. According to the American Joint Committee on Cancer (AJCC) staging manual, patients with satellite/in-transit metastasis and/or regional lymph node disease are classified as stage III melanoma, which is further divided into subgroups according to prognosis. The AJCC 7th edition was recently replaced with the updated AJCC 8th edition, and the number of subgroups in stage III were revised from three $(\mathrm{A}-\mathrm{C})$ to four $(\mathrm{A}-\mathrm{D})$ groups. ${ }^{1}$ The subgroup classification now depends on both $\mathrm{T}$ and $\mathrm{N}$ status, and the previous low-risk group (stage IIIA) has an even better prognosis, while patients with the highest risk are now classified as stage IIID. This substage migration can have an impact on the clinical management of patients with stage III melanoma.
ASO Author Reflections is a brief invited commentary on the article "A Population-Based Comparison of the AJCC 7th and AJCC 8th Editions for Patients Diagnosed with Stage III Cutaneous Malignant Melanoma in Sweden”, Ann Surg Oncol. 2019;26:2839-2845.

(C) Society of Surgical Oncology 2019

First Received: 8 August 2019;

Published Online: 29 August 2019

R. Olofsson Bagge, MD

e-mail: roger.olofsson@gu.se

\section{PRESENT}

Currently, there is a paradigm shift with the introduction of new effective systemic treatments for patients with both stage III and IV disease. Three immune checkpoint inhibitors have been approved for use in advanced melanoma, i.e. the CTLA- 4 inhibitor ipilimumab and the PD-1 inhibitors nivolumab and pembrolizumab. In addition, targeted therapies (BRAF/MEK inhibitors) are also available as a treatment for patients with BRAF-mutated melanomas. Adjuvant trials have recently shown a relapse-free survival (RFS) benefit of systemic treatment in stage III melanoma, leading to a change in therapeutic approach for stage III patients after radical surgery; ${ }^{2}$ however, all these adjuvant trials were performed using the former AJCC 7th edition for stage classification. A recent publication using a prospective population-based registry including all Swedish patients with melanoma showed an improved 5- and 10-year melanoma-specific survival (MSS) for substages IIIA, IIIB, and IIIC when patients were reclassified according to the updated AJCC 8th edition, while the new substage IIID had the worst prognosis. ${ }^{3}$

\section{FUTURE}

A recent update from one of the adjuvant trials (the EORTC1325/KEYNOTE-054 trial) showed that only $8 \%$ of patients would be classified as stage IIIA according to the AJCC 8th edition, compared with $15 \%$ according to the AJCC 7th edition used in the trial. Moreover, this analysis showed that when reclassifying patients according to the AJCC 8th edition, the 1-year recurrence-free survival in the stage IIIA subgroup was $92.7 \%$ versus $92.5 \%$ in the placebo arm. ${ }^{4}$ These differences based on substage migration 
with the new AJCC staging are very important when considering the effects of adjuvant therapies. To date, survival data are only available for the adjuvant use of ipilimumab. In this trial, there was an overall survival benefit at 5-years, with a hazard ratio of 0.72 in favor of ipilimumab compared with placebo for all stage III patients. ${ }^{5}$ When considering this relative risk on the current data presented for the Swedish stage IIIA population, the absolute gain in survival at 5 years would be $6.4 \%$ using the AJCC 7th edition, compared with $3.6 \%$ using the AJCC 8th edition, increasing the number needed to treat from approximately $17-25$ patients. ${ }^{3}$ These findings highlight the importance of analysing new adjuvant melanoma trials in light of the new AJCC 8th edition, especially when overall survival data for the modern adjuvant trials are published.

DISCLOSURES Roger Olofsson Bagge has received research grants from AstraZeneca, speaker honorarium from Roche and Pfizer, and has served on advisory boards for Amgen, BMS, and MSD (the advisory boards for BMS and MSD related to adjuvant treatment of melanoma). Dimitrios Katsarelias and Karolin Isaksson have no conflicts of interest to declare.

\section{REFERENCES}

1. Gershenwald JE, Scolyer RA, Hess KR, Sondak VK, Long GV, Ross MI, et al. Melanoma staging: Evidence-based changes in the American Joint Committee on Cancer eighth edition cancer staging manual. CA Cancer J Clin. 2017;67(6):472-92.

2. Blankenstein SA, van Akkooi ACJ. Adjuvant systemic therapy in high-risk melanoma. Melanoma Res. 2019;29(4):358-64.

3. Isaksson K, Katsarelias D, Mikiver R, Carneiro A, Ny L, Olofsson Bagge R. A Population-Based Comparison of the AJCC 7th and AJCC 8th Editions for Patients Diagnosed with Stage III Cutaneous Malignant Melanoma in Sweden. Ann Surg Oncol. 2019; 26:2839-45.

4. Eggermont AMM, Blank CU, Mandala M, Long GV, Atkinson VG, Dalle S, et al. Prognostic and predictive value of AJCC-8 staging in the phase III EORTC1325/KEYNOTE-054 trial of pembrolizumab vs placebo in resected high-risk stage III melanoma. Eur J Cancer. 2019;116:148-57.

5. Eggermont AM, Chiarion-Sileni V, Grob JJ, Dummer R, Wolchok JD, Schmidt H, et al. Prolonged Survival in Stage III Melanoma with Ipilimumab Adjuvant Therapy. $\mathrm{N}$ Engl $\mathrm{J}$ Med. 2016;375(19):1845-55.

Publisher's Note Springer Nature remains neutral with regard to jurisdictional claims in published maps and institutional affiliations. 